\title{
RIGHLIGHTS
}

CNS CANCER

\section{CSF DNA provides a snapshot of the glioma genome}

Genetic profiling is increasingly being used for disease classification and treatment allocation in patients with diffuse gliomas, which include glioblastoma and lower-grade gliomas. Owing to the location of these tumours, tissue sampling is challenging, particularly repeat sampling aimed at capturing disease evolution following therapy. Liquid biopsy sampling provides new opportunities in this regard, but the scope for blood-based detection of DNA derived from central nervous system (CNS) cancers might be limited by the blood-brain barrier. Now, a study reported in Nature highlights the promise of genetic profiling of gliomas via liquid biopsy of cerebrospinal fluid (CSF).

"Several studies have confirmed that tumour DNA can be detected in CSF from patients with CNS cancers, but these studies often included low numbers of patients with different types of brain tumours, were focused of the detection of specific lesions known to exist in the tumour from the same patient and used CSF samples collected during surgery," says Ingo Mellinghoff. "Our study distinguishes itself from prior studies by its exclusive focus on diffuse glioma, the larger number of patients examined, the detailed clinical and radiographic annotation of each patient, the comprehensive nature of our nextgeneration sequencing (NGS) test and the method of CSF collection (spinal tap)," he adds.

CSF samples collected from 85 patients who presented with new neurological symptoms following treatment of diffuse glioma were probed for mutations, copy number alterations and chromosomal aberrations using the FDAauthorized MSK-IMPACT assay.
"Because this assay has already been extensively validated using tumour biopsy samples, we could interpret the results without any prior tumour profiling," Mellinghoff explains.

Tumour DNA was detected in the CSF of 42 patients (49.4\%) and harboured many alterations that are typical for glioma, including 1p/19q co-deletions and IDH1, TP53, ATRX and/or TERT promoter mutations, as well as CDKN2A and/or CDKN2B deletions and EGFR aberrations. Only 3 (16\%) of 19 patients with mutations detected in CSF DNA had a mutation detected in plasma cell-free DNA using a high-sensitivity, but admittedly more targeted, capture-based NGS assay.

Notably, radiographic findings, including tumour progression, burden and pattern of spread, were correlated with the detection of tumour-derived DNA in CSF, thus indicating the prognostic value of the test. Indeed, on multivariate analysis, patients with tumour DNA detected in CSF had a greater risk of death than those without detectable CSF tumour DNA $(P<0.000025)$.

Molecular alterations that are used in the WHO classification of gliomas, such as $I D H$ mutations and $1 \mathrm{p} / 19 \mathrm{q}$ co-deletions, were highly concordant between CSF and matched tumour samples (with $100 \%$ concordance in 30 patients without DNA hypermutation), and these subtypedefining mutations persisted throughout the disease course.

In general, mutation profiles were found to be highly concordant between CSF replicates $(n=6)$ or CSF-tumour sample pairs $(n=5)$ collected within 3 weeks of each other. Thus, "CSF tumour DNA provides an accurate 'snapshot' of the tumour genome at the time

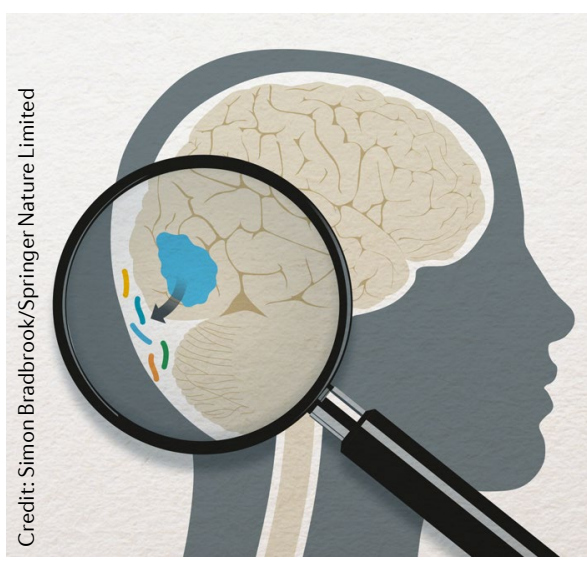

of CSF collection," states Michael Berger, who co-led the study. In the overall group of 36 patients with paired tumour DNA-positive CSF and tumour samples, the percentage of shared mutations was also high, although concordance was typically lower for subclonal mutations and decreased with increasing intervals between tumour and CSF sampling. Of note, many alterations in growth factor signalling pathways changed dynamically during the disease course. These findings indicate that analyses of CSF DNA can uncover patterns of tumour evolution, with potential implications for therapy.

"Our approach might help us obtain a comprehensive genomic profile in patients with tumours that cannot be biopsied owing to their location," Berger explains. "Having a more tractable method to re-evaluate the cancer genome will also enable us to better match treatments with specific genetic alterations and help us understand the mechanisms of resistance to targeted therapies," he concludes. The investigators plan to incorporate their liquid biopsy test into new strategies for the treatment of glioma and are also testing their assay in patients with other CNS cancers, such as CNS lymphoma.

David Killock

ORIGINAL ARTICLE Miller, A. M. et al. Tracking tumour evolution in glioma through liquid biopsies of cerebrospinal fluid. Nature 565, 654-658 (2019) 